Provided for non-commercial research and education use. Not for reproduction, distribution or commercial use.

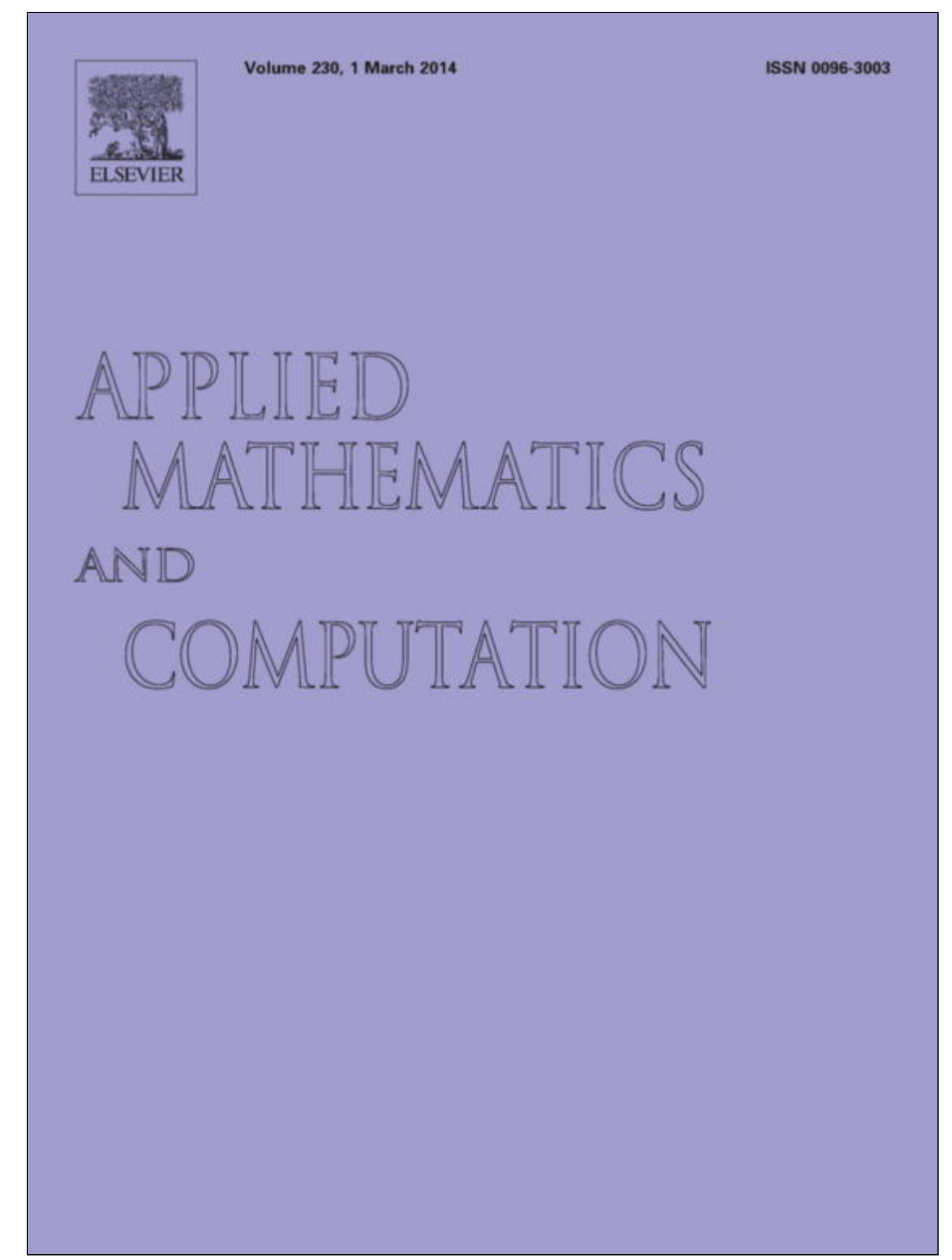

This article appeared in a journal published by Elsevier. The attached copy is furnished to the author for internal non-commercial research and education use, including for instruction at the authors institution and sharing with colleagues.

Other uses, including reproduction and distribution, or selling or licensing copies, or posting to personal, institutional or third party websites are prohibited.

In most cases authors are permitted to post their version of the article (e.g. in Word or Tex form) to their personal website or institutional repository. Authors requiring further information regarding Elsevier's archiving and manuscript policies are encouraged to visit:

http://www.elsevier.com/authorsrights 


\title{
Quadratic model of inter-population interaction: Investigation of stability areas
}

\author{
V.Ye. Belozyorov ${ }^{\mathrm{a}, *}$, S.V. Chernyshenko ${ }^{\mathrm{b}}$ \\ a Department of Applied Mathematics, Dnepropetrovsk National University, Gagarin's Avenue, 72, 49050 Dnepropetrovsk, Ukraine \\ ${ }^{\mathrm{b}}$ Faculty of Computer Sciences, Institute of Information Systems, University of Koblenz-Landau, University Street 1, D-56070 Koblenz, Germany
}

\section{A R T I C L E I N F O}

\section{Keywords:}

System of ordinary quadratic differential equations

Asymptotic stability cone

Complete linear group

Invariant

Biological population

\begin{abstract}
A B S T R A C T
A development quadratic model of the heterogeneous biological population consisting of a few sub-populations is investigated. The classic stabilization problem of solutions for the system of ordinary quadratic differential equations, which describing this model, is solved. Examples are given.
\end{abstract}

(c) 2013 Elsevier Inc. All rights reserved.

\section{Introduction}

The logistic equation

$$
\dot{x}(t)=a x(t)\left(1-\frac{x(t)}{k}\right), \quad x(t) \geqslant 0, a>0, k>0
$$

is a very classical (but still actual) tool for description of population growth [1]. The equation is based on the use of two fundamental population characteristics: $a$ is the coefficient of productivity, which characterizes potential intensity of population's growth, and $k$ is the"environment volume". This coefficient reflects an equilibrium size of the population, which can be realized in considering environment. Naturally, the description of a population by two positive real numbers is an essential simplification. Nevertheless this simplification is quite useful and popular [2,3].

One of factors, which are not taken into account in the model (1), is heterogeneity of populations. It is clear that different specimens have different abilities, both of growth rate and potential density. In (1) one considers average values; it is quite correct if there is some normal distribution of the indices. The real situation is usually more complex: a population can be divided for some more or less discrete groups (sub-populations) and indices within each of them are more similar each other that between the groups. Forming the population these groups are not completely isolated; it is very important that they can "exchange" by specimens (both directly and through reproduction).

Each $i$ th subpopulation is still characterized by one value $k_{i}(i=1, \ldots, n ; n$ is a number of the groups) which means stable size of the subpopulation in the environment (in the case of absence of other sub-populations!). In the same time the growth coefficient should reflect possibility of production by this subpopulation of new members for other sub-populations. One should consider a number of $a_{i j}(i, j=1, \ldots, n)$, which reflects an intensity of production of specimens of $j$ th subpopulation by $i$ th one $(i \neq j)$. The coefficient $a_{i i} ; i=1, \ldots, n$, describes the growth ("self-support") of $i$ th subpopulation. It is quite logical to propose for the description of general dynamics of the heterogeneous biological population the following system equations:

\footnotetext{
* Corresponding author.

E-mail addresses: belozvye@mail.ru (V.Ye. Belozyorov), svc@a-teleport.com (S.V. Chernyshenko).
} 


$$
\left\{\begin{array}{l}
\dot{x}_{1}(t)=\sum_{j=1}^{n} a_{1 j} x_{j}(t)\left(1-\frac{x_{j}(t)}{k_{j}}\right), \\
\ldots \ldots \ldots \ldots \ldots \ldots \ldots \ldots \ldots \ldots \ldots \ldots \ldots \ldots \\
\dot{x}_{n}(t)=\sum_{j=1}^{n} a_{n j} x_{j}(t)\left(1-\frac{x_{j}(t)}{k_{j}}\right) .
\end{array}\right.
$$

Here $x_{i}(t)$ is a vector of states; the vector of initial values $\mathbf{x}^{T}(0)=\left(x_{10}, \ldots, x_{n 0}\right)$ is given; $a_{i j}$ and $k_{j}>0$ are real numbers; $i, j=1, \ldots, n$.

Nature of the sub-populations can be quite different. They can be real sub-populations with genetic peculiarities and essentially isolated geographically (and in this case the diagonal coefficients $a_{i i}$ will be much bigger then other ones). System (2) can also describe intensive interaction between representatives of different genetic lines, which can take place as a permanent component of population dynamics [4]. As example of such process one can mention interaction between human races or a change of quantities of blondes and brunettes in the human population. The model can be applied for any kind of heterogeneity, but in some case (for example, for age heterogeneity) it is transformed to very special degenerate kind.

Another possibility it is't consider genetic, but "social" groups. An existence of "horizontal" hierarchy (a separation of populations for families, bevies and so on) means the existence of "vertical" one (there are "social classes" of specimens: ordinary specimens, heads of family, bevy leaders) [5]. Contrary to sex difference specimens can change their group membership, although this change is not as mechanistic as change of age. Big populations of "intellectual" animals ( particularly primates, ungulates, cetaceans, and even insects and fishes [6,7]) often form multi-level social structures. There are quite fundamental descriptions of social structures of several special species [8,9]. In some cases (for example, for Galapagos sea-lion [9]) it was even possible to estimate the number of layers in population social structure. Number of the levels depends on their both intellectual and "energetic" abilities [5].

Although model (2) has quite evident form and mentioned in scientific literature [10-12], it is not deeply discussed and investigated. Partially, it can be explained by quit high level of its mathematical complexity and big number of its parameters. Some first steps of investigation of the model was done in paper [13], where model (2) was called as "zygote elimination model" (several group of cells were considered as sub-populations; it is another opportunity to interpret the model). In current article we want to continue the investigation on quite formal mathematical level.

Let $x_{10}>0, \ldots, x_{n 0}>0$. Then generally speaking there exists a finite time $t^{*}$ and $i \in\{1, \ldots, n\}$ such that $x_{i}\left(t^{*}\right)<0$. If the last inequality takes place, then from the biological point of view model (2) is improper. Nevertheless if $\forall t>0$, we have $x_{i}(t)>0 ; i=1, \ldots, n$, then model (2) can correctly describe the dynamic behavior of some heterogeneous biological population.

In [14-16] the existence conditions of the asymptotic stability cone for general quadratic systems were resulted. However these conditions have a local character. A main task of the present paper is constructing of a global stability region for system (2).

\section{Preliminary results}

In this section we remind some known results which are resulted in works [14-16].

Consider the homogeneous quadratic system of order $n$ :

$$
\left\{\begin{array}{c}
\dot{x}_{1}(t)=\mathbf{x}^{T}(t) B_{1} \mathbf{x}(t), \\
\cdots \cdots \cdots \cdots \cdots \cdots \cdots \\
\dot{x}_{n}(t)=\mathbf{x}^{T}(t) B_{n} \mathbf{x}(t)
\end{array}\right.
$$

with the vector of initial values $\mathbf{x}^{T}(0)$. Matrices $B_{1}, \ldots, B_{n} \in \mathbb{R}^{m \times m}$ are real and symmetrical.

Notice that any quadratic form in the right-hand side of system (3) can be written as

$$
\mathbf{x}^{T} B_{i} \mathbf{x}=\left(\mathbf{r}_{i 1}, \ldots, \mathbf{r}_{i n}\right) \cdot\left(x_{1} \mathbf{x}^{T}, \ldots, x_{n} \mathbf{x}^{T}\right)^{T}
$$

where $\mathbf{r}_{i 1}, \ldots, \mathbf{r}_{i n} \in \mathbb{R}^{\mathrm{m}}$ are row-vectors of the matrix $B_{i}, i=1, \ldots, n$. Thus, any system (3) can be presented by the such form

$$
\dot{\mathbf{x}}(t)=\mathbf{T} \cdot(\mathbf{x}(t) \otimes \mathbf{x}(t))
$$

where

$$
\mathbf{T}=\left(\begin{array}{ccc}
\mathbf{r}_{11}, & \cdots & , \mathbf{r}_{1 n} \\
\vdots & \ldots & \vdots \\
\mathbf{r}_{n 1}, & \cdots & , \mathbf{r}_{n n}
\end{array}\right) \in \mathbb{R}^{n \times n^{2}}
$$

and $\mathbf{T}$ is a mixed tensor of rank 3 (once contravalent and twice covalent); $\mathbf{x} \otimes \mathbf{x}=\left(x_{1} \mathbf{x}^{T}, \ldots, x_{n} \mathbf{x}^{T}\right)^{T}$ is a tensor product of the vector $\mathbf{x}$ with itself. (Here the tensor $\mathbf{T}$ is realized as an element of the space matrices of sizes $n \times n^{2}$.) 
The tensor $\mathbf{T}$ can be presented by matrices $\mathbf{T}=\left(T_{1}, \ldots, T_{n}\right)$, where

$$
T_{1}=\left(\begin{array}{c}
\mathbf{r}_{11} \\
\vdots \\
\mathbf{r}_{n 1}
\end{array}\right) \in \mathbb{C}^{n \times n}, \ldots, T_{n}=\left(\begin{array}{c}
\mathbf{r}_{1 n} \\
\vdots \\
\mathbf{r}_{n n}
\end{array}\right) \in \mathbb{C}^{n \times n}
$$

Define the function (it is an invariant of the complete linear group $\mathbb{G} \mathbb{L}(n, \mathbb{C})[14]$ ):

$$
I\left(T_{1}, \ldots, T_{n}\right)=\operatorname{det}\left(\sum_{1 \leqslant j_{1}, \ldots, j_{n} \leqslant n}(-1)^{\sigma} T_{j_{1}} T_{j_{2}} \cdot \ldots \cdot T_{j_{n}}\right),
$$

where $\sigma=0$, if a permutation $\left(j_{1}, \ldots, j_{n}\right)$ is even and $\sigma=1$, if the permutation $\left(j_{1}, \ldots, j_{n}\right)$ is odd.

Definition 1. System (3) (or (4)) is called a regular if $I\left(T_{1}, \ldots, T_{n}\right) \neq 0$.

The importance of concept of the regularity will be shown below.

We return to system (2). Fulfill the following identical transformations:

$$
x_{j}\left(1-\frac{x_{j}}{k_{j}}\right) \equiv \frac{k_{j}}{4}-\left(\frac{x_{j}}{\sqrt{k_{j}}}-\frac{\sqrt{k_{j}}}{2}\right)^{2} ; j=1, \ldots, n .
$$

Introduce new variables $y_{j}$ under the formulas:

$$
y_{j}=\frac{x_{j}}{\sqrt{k_{j}}}-\frac{\sqrt{k_{j}}}{2} ; j=1, \ldots, n .
$$

Then in variables $y_{j}$ system (2) can be represented in the form

$$
\dot{\mathbf{y}}(t)=\mathbf{b}-C \mathbf{y}^{2}(t) \equiv\left\{\begin{array}{l}
\dot{y}_{1}(t)=b_{1}-\sum_{j=1}^{n} c_{1 j} y_{j}^{2}(t), \\
\ldots \ldots \ldots \ldots \ldots \cdots, \ldots \ldots \cdots \cdots \\
\dot{y}_{n}(t)=b_{n}-\sum_{j=1}^{n} c_{n j} y_{j}^{2}(t),
\end{array}\right.
$$

where $\mathbf{y}=\left(y_{1}, \ldots, y_{n}\right)^{T}, \mathbf{y}^{2}=\left(y_{1}^{2}, \ldots, y_{n}^{2}\right)^{T}$,

$$
\begin{aligned}
& \mathbf{b}=\left(\begin{array}{c}
b_{1} \\
\vdots \\
b_{n}
\end{array}\right)=\left(\begin{array}{cccc}
\left(k_{1}\right)^{-1 / 2} / 4 & \ldots & 0 \\
& & \ddots & \vdots \\
& & \ldots & \left(k_{n}\right)^{-1 / 2} / 4
\end{array}\right) \cdot\left(\begin{array}{ccc}
a_{11} & \ldots & a_{1 n} \\
\vdots & \ddots & \vdots \\
a_{n 1} & \ldots & a_{n n}
\end{array}\right) \cdot\left(\begin{array}{c}
k_{1} \\
\vdots \\
k_{n}
\end{array}\right), \\
& C=\left(\begin{array}{ccc}
\left(k_{1}\right)^{-1 / 2} & \ldots & 0 \\
\vdots & \ddots & \vdots \\
0 & \ldots & \left(k_{n}\right)^{-1 / 2}
\end{array}\right) \cdot\left(\begin{array}{ccc}
a_{11} & \ldots & a_{1 n} \\
\vdots & \ddots & \vdots \\
a_{n 1} & \ldots & a_{n n}
\end{array}\right) .
\end{aligned}
$$

Alongside with system (6), we will consider the system of ordinary quadratic differential equations

$$
\dot{\mathbf{y}}(t)=-C \mathbf{y}^{2}(t) \text {. }
$$

Introduce the matrices:

$$
T_{i}=\left(\begin{array}{ccccccc}
0 & \ldots & 0 & c_{1 i} & 0 & \ldots & 0 \\
\vdots & \vdots & \vdots & \vdots & \vdots & \vdots & \vdots \\
0 & \ldots & 0 & c_{n i} & 0 & \ldots & 0
\end{array}\right) \in \mathbb{R}^{n \times n} ; \quad i=1, \ldots, n .
$$

Lemma 1. If $I\left(T_{1}, \ldots, T_{n}\right) \neq 0$, then $\operatorname{det} C \neq 0$.

Proof. An elementary check shows that the following equality takes place:

$$
\sum_{1 \leqslant j_{1}, \ldots j_{m} \leqslant n}(-1)^{\sigma} T_{j_{1}} T_{j_{2}} \cdot \ldots \cdot T_{j_{m}}=C \cdot F(C),
$$

where $F(C)=\left(f_{i j}(C)\right)$ is $(n \times n)$-matrix, coefficients of which depend on coefficients of the matrix $C ; f_{i i}(C)=0, i=1, \ldots, n$. Hence $I\left(T_{1}, \ldots, T_{n}\right)=\operatorname{det} C \cdot \operatorname{det} F(C)$ and if $I\left(T_{1}, \ldots, T_{n}\right) \neq 0$, then $\operatorname{det} C \neq 0$. 
For example, for $n=2 I\left(T_{1}, T_{2}\right)=c_{12} c_{21} \operatorname{det} C$. If $n=3$, then $I\left(T_{1}, T_{2}, T_{3}\right)=\left(\left(c_{12} c_{23} c_{31}\right)^{2}-\left(c_{13} c_{21} c_{32}\right)^{2}\right) \operatorname{det} C$.

Let system (7) be the regular. Using Lemma 1 , we transform this system in the following way. Introduce variables $w_{1}, \ldots, w_{n}$ under the formula

$$
\mathbf{w}=\left(\begin{array}{c}
w_{1} \\
\vdots \\
w_{n}
\end{array}\right)=C^{-1} \cdot\left(\begin{array}{c}
y_{1} \\
\vdots \\
y_{n}
\end{array}\right) \equiv C^{-1} \mathbf{y}
$$

Then system (6) can be written as

$$
\dot{\mathbf{w}}(t)=C^{-1} \mathbf{b}-\left(\begin{array}{c}
\left(c_{11} w_{1}(t)+\cdots+c_{1 n} w_{n}(t)\right)^{2} \\
\vdots \\
\left(c_{n 1} w_{1}(t)+\cdots+c_{n n} w_{n}(t)\right)^{2}
\end{array}\right)=\frac{1}{4}\left(\begin{array}{c}
k_{1} \\
\vdots \\
k_{n}
\end{array}\right)-\left(\begin{array}{c}
\left(d_{11} w_{1}(t)+\cdots+d_{1 n} w_{n}(t)\right)^{2} \\
\vdots \\
\left(d_{n 1} w_{1}(t)+\cdots+d_{n n} w_{n}(t)\right)^{2}
\end{array}\right),
$$

where $d_{i j}=a_{i j} / \sqrt{k_{i}}$ and system (7) can be written as

$$
\dot{\mathbf{w}}(t)=-\left(\begin{array}{c}
\left(d_{11} w_{1}(t)+\cdots+d_{1 n} w_{n}(t)\right)^{2} \\
\vdots \\
\left(d_{n 1} w_{1}(t)+\cdots+d_{n n} w_{n}(t)\right)^{2}
\end{array}\right) .
$$

Definition 2. We call (3) a regular $\mathbb{R}$-system if:

(1) system (3) is the regular;

(2) there exists a real nonsingular matrix $S=\left(s_{i j}\right) \in \mathbb{R}^{n \times n}$ such that the form $\mathbf{x}^{T}\left(s_{i 1} B_{1}+\cdots+s_{i n} B_{n}\right) \mathbf{x}$ is negative for all $i=1, \ldots, n$.

Let (3) be a regular $\mathbb{R}$-system. Then there is a nonsingular matrix $S \in \mathbb{R}^{n \times n}$ such that variables $\mathbf{w}(t)=S^{-1} \mathbf{x}(t)=\left(w_{1}(t), \ldots, w_{n}(t)\right)^{T}$ satisfy by equation

$$
\left(\begin{array}{c}
\dot{w}_{1}(t) \\
\vdots \\
\dot{w}_{n}(t)
\end{array}\right)=S^{-1}\left(\begin{array}{c}
(S \mathbf{w}(t))^{T} B_{1}(S \mathbf{w}(t)) \\
\vdots \\
(S \mathbf{w}(t))^{T} B_{n}(S \mathbf{w}(t))
\end{array}\right)=-\left(\begin{array}{c}
(\mathbf{w}(t))^{T} D_{1} \mathbf{w}(t) \\
\vdots \\
(\mathbf{w}(t))^{T} D_{n} \mathbf{w}(t)
\end{array}\right),
$$

where $D_{i} \subset \mathbb{R}^{n \times n}$ are symmetrical matrices and all quadratic forms $-\mathbf{w}^{T} D_{i} \mathbf{w}, i=1, \ldots, n$, are negative. (Thus, system (9) is the regular $\mathbb{R}$-system.)

Notice that by small perturbations of the matrix $S$ it is possible to obtain that for the vector of initial data $\left(w_{10}, \ldots, w_{n 0}\right)^{T}$ of system (10) the condition $w_{n 0} \neq 0$ will be fulfilled. In addition, the regularity of system (10) will be preserved. Further, we will consider that $w_{n 0} \neq 0$.

Let $z_{1}=w_{1} / w_{n}, \ldots, z_{n-1}=w_{n-1} / w_{n}$. Then from (10) it follows that

$$
\left(\begin{array}{c}
\dot{z}_{1}(t) \\
\vdots \\
\dot{z}_{n-1}(t) \\
\dot{w}_{n}(t)
\end{array}\right)=\left(\begin{array}{c}
\frac{\dot{w}_{1} w_{n}-w_{1} \dot{w}_{n}}{w_{n}^{2}} \equiv G_{1}\left(z_{1}(t), \ldots, z_{n-1}(t)\right) w_{n}(t) \\
\ldots \ldots \ldots \ldots \ldots \ldots \ldots \ldots \ldots \ldots \\
\frac{\dot{w}_{n-1} w_{n}-w_{n-1} \dot{w}_{n}}{w_{n}^{2}} \equiv G_{n-1}\left(z_{1}(t), \ldots, z_{n-1}(t)\right) w_{n}(t) \\
G_{n}\left(z_{1}(t), \ldots, z_{n-1}(t)\right) w_{n}^{2}(t)
\end{array}\right)
$$

where $G_{i}\left(z_{1}, \ldots, z_{n-1}\right)$ are inhomogeneous cubic functions and $G_{n}\left(z_{1}, \ldots, z_{n-1}\right)$ is an inhomogeneous negative quadratic function with respect to $z_{1}, \ldots, z_{n-1}$.

Consider the following equations

$$
\left\{\begin{array}{l}
G_{1}\left(z_{1}, \ldots, z_{n-1}\right) \equiv z_{1} \cdot\left(\mathbf{z}^{T}, 1\right) D_{n}\left(\mathbf{z}^{T}, 1\right)^{T}-\left(\mathbf{z}^{T}, 1\right) D_{1}\left(\mathbf{z}^{T}, 1\right)^{T}=0, \\
\ldots \ldots \ldots \ldots \ldots \ldots \ldots \ldots \ldots \\
G_{n-1}\left(z_{1}, \ldots, z_{n-1}\right) \equiv z_{n-1} \cdot\left(\mathbf{z}^{T}, 1\right) D_{n}\left(\mathbf{z}^{T}, 1\right)^{T}-\left(\mathbf{z}^{T}, 1\right) D_{n-1}\left(\mathbf{z}^{T}, 1\right)^{T}=0 .
\end{array}\right.
$$

Denote by $\mathbf{z}_{1}^{*}, \ldots, \mathbf{z}_{l}^{*} \in \mathbb{R}^{n-1}, 1 \leqslant l \leqslant 2^{n}-1$, all real solutions of system (12). (It is clear that any real solution of system (12) is positive.) It is known [14,15] that system (12) in the regular case has exactly $2^{n}-1$ (it is odd number) complex solutions. Consequently the real solutions exist. 
Notice that system (12) is equivalent to the algebraic system

$$
\left\{\begin{array}{c}
w_{1}=-\mathbf{w}^{T} D_{1} \mathbf{w} \\
\cdots \cdots \cdots \cdots \\
w_{n}=-\mathbf{w}^{T} D_{n} \mathbf{w}
\end{array}\right.
$$

in such sense: any nonzero solution $\mathbf{w}^{*}$ of system (13) is connected with the solution $\mathbf{z}^{*}$ of system (12) by the formula

$$
\mathbf{w}^{*}=\left(w_{1}^{*}, \ldots, w_{n-1}^{*}, w_{n}^{*}\right)=w_{n}^{*} \cdot\left(\mathbf{z}^{* T}, 1\right)=w_{n}^{*} \cdot\left(z_{1}^{*}, \ldots, z_{n-1}^{*}, 1\right) ; w_{n}^{*} \neq 0 .
$$

Let $\mathbb{W} \subset \mathbb{C}^{n}$ be an algebraic variety of all solutions of system (13) [17].

Definition 3. System (13) is called complete if $\operatorname{deg}_{\mathbb{C}} \mathbb{W}=\operatorname{deg}_{\mathbb{C}} \mathbb{L}$, where $\mathbb{L} \subset \mathbb{R}^{n}$ is the variety of all solutions of system (13), for which it is supposed that all elements of matrices $B_{1}, \ldots, B_{n}$ are not numbers but independent parameters.

In $[14,15]$ are proved that the regular system $(13)$ is complete and $\operatorname{deg}_{\mathbb{C}} \mathbb{W}=2^{n}-1$. Exactly on this fact all further reasonings are built.

For example, let's consider system (7) for $n=2$. If $I\left(T_{1}, T_{2}\right) \neq 0$, then $\operatorname{deg}_{\mathbb{C}} \mathbb{W}=2^{2}-1=3$; if $I\left(T_{1}, T_{2}\right)=0$, then in this case $\operatorname{deg}_{\mathbb{C}} \mathbb{W} \leqslant 2$.

\section{An asymptotic stability cone of homogeneous quadratic system}

Definition 4. A closed domain $\Theta \subset \mathbb{R}^{n}$ containing the origin is called a cone if for all $\mathbf{x} \in \Theta$ and all $\lambda \geqslant 0$ we have $\lambda \mathbf{x} \in \Theta$. (Generators of this cone are the rays starting from the origin.) A cone is said to be trivial if there exists a proper subspace $\mathcal{D} \subset \mathbb{R}^{n}$ such that $\mathcal{D} \subseteq \Theta$.

Denote by $\mathbf{x}\left(\mathbf{x}_{0}, t\right)$ the solution of system (3) with a vector of initial values $\mathbf{x}_{0}$.

Definition 5. The trivial solution $\mathbf{x} \equiv 0$ of system (3) is said to be conditionally asymptotically stable with respect to the nontrivial cone $\Theta$ if for all $\mathbf{x}_{0} \in \Theta$ the following conditions hold:

(a) $\left\|\mathbf{x}\left(\mathbf{x}_{0}, t\right)\right\|<M$ for all $t \geqslant 0$, where $M>0$ is some constant;

(b) $\lim _{t \rightarrow \infty}\left\|\mathbf{x}\left(\mathbf{x}_{0}, t\right)\right\|=0$.

In this case, $\Theta$ is called an asymptotic stability cone.

We designate by $\mathbb{R}^{n+}$ an orthant nonnegative coordinates (it is a cone) in the space $\mathbb{R}^{n}$ and we also designate by $\mathbb{R} \subset \mathbb{R}^{n+}$ an asymptotic stability cone of system (10). (A vertex of this cone coincides with original of coordinates.)

Systems (10) were studied in [14-16]. However the geometry of the cone $\mathbb{K}$ was not investigated. The present work is called to make clear a structure of this cone. Besides, it will be shown applications obtained results to system (2).

Let $\mathbb{P}_{i}$ be a set all real solutions of equation $G_{i}\left(z_{1}, \ldots, z_{n-1}\right)=0, i \in\{1, \ldots, n-1\}$. (This is a surface in $\mathbb{R}^{n-1}$.).

Consider a decomposition of the space $\mathbb{R}^{n-1}$ by surfaces $\mathbb{P}_{1}, \ldots, \mathbb{P}_{n-1}$ on regions $\Sigma_{i}, i=1, \ldots, p$.

Denote by $\bar{\Sigma}_{1}=\Sigma_{1} \cup S_{1}, \ldots, \bar{\Sigma}_{p}=\Sigma_{p} \cup \mathbb{S}_{p}$ closed regions in $\mathbb{R}^{n-1}$ of the same dimension $n-1$ (Fig. 1 ). The boundaries $\mathbb{S}_{1}, \ldots, \mathbb{S}_{p}$ of regions $\bar{\Sigma}_{1}, \ldots, \bar{\Sigma}_{p}$ are pieces of surfaces $\mathbb{P}_{1}, \ldots, \mathbb{P}_{n-1}$ which describe by Eqs. (12). Thus, here boundaries $\mathbb{S}_{i}, i=1, \ldots, p$, are $(n-2)$-dimensional varieties. Note that the number $p$ of these boundaries generally speaking is more than number $n-1$ of Eqs. (12); it means that each of Eqs. (12) can represent a few boundaries.

Without loss of generality, we can consider that

$$
\mathbb{R}^{n-1}=\bar{\Sigma}_{1} \cup \cdots \cup \bar{\Sigma}_{p} \text { and if } i \neq j, \quad \text { then } \Sigma_{i} \cap \Sigma_{j}=\emptyset .
$$

It is clear that for any real solution $\mathbf{z}_{k}^{*}, k \in\{1, \ldots, l\}$, of system (12) there exists exactly $r=2^{n-1} \leqslant p$ regions $\bar{\Sigma}_{j_{1}}, \ldots, \bar{\Sigma}_{j_{r}}, j_{1}, \ldots j_{r} \in\{1, \ldots, p\}$, such that $\mathbf{z}_{k}^{*}=\bar{\Sigma}_{j_{1}} \cap \cdots \cap \bar{\Sigma}_{j_{r}}$.

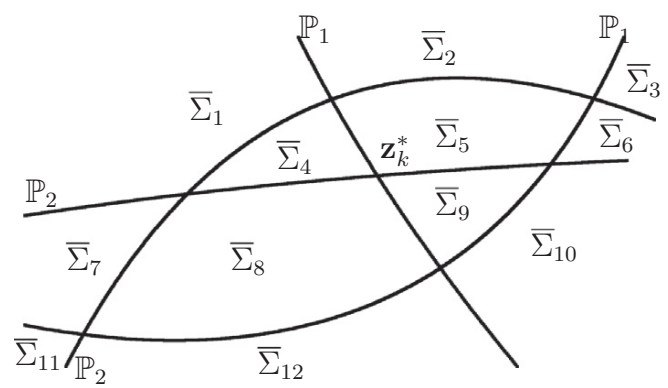

Fig. 1. The graphic interpretation of the region $\bar{\Sigma}$ for $n=3$. 
Denote by $\bar{\Sigma}=\bar{\Sigma}_{j_{1}} \cup \cdots \cup \bar{\Sigma}_{j_{r}}$. (Generally speaking the region $\bar{\Sigma}$ may be unbounded.) For example, let $n=3, p=12, r=2^{n-1}=4, j_{1}=4, j_{2}=5, j_{3}=8$, and $j_{4}=9$. Then for the point $\mathbf{z}_{k}^{*}$ we have

$$
\bar{\Sigma}=\bar{\Sigma}_{4} \cup \bar{\Sigma}_{5} \cup \bar{\Sigma}_{8} \cup \bar{\Sigma}_{9}
$$

In Fig. 1 it is shown the typical placement of regions $\bar{\Sigma}_{i}, i=1, \ldots, 12$, in the case $n=3$. (Here $\bar{\Sigma}$ is bounded.)

There are two lines $\mathbb{P}_{1}$ and $\mathbb{P}_{2}$ which form the boundaries of the region $\bar{\Sigma}_{i}, i=1, \ldots, 12$. For example, the boundary of the region $\bar{\Sigma}_{8}$ composes from 4 pieces of lines $\mathbb{P}_{1}$ and $\mathbb{P}_{2}$.

Let $\mathbb{R}^{n+}$ be an orthant of nonnegative coordinates. Denote by $\mathbb{K}=\{x \bar{\Sigma}, x\} \subset \mathbb{R}^{n+}$ a cone that has been constructed for the region $\bar{\Sigma}$ with a vertex in original of coordinates. (Here $x$ is passing the set of nonnegative numbers.)

Geometrically the cone $\mathbb{K}$ can be defined as follows.

In the system coordinates $\left(w_{1}, \ldots, w_{n}\right)$ we construct a plane $w_{n}=1$ in parallel by the coordinate plane $\left(w_{1}, \ldots, w_{n-1}\right)$. Then the region $\bar{\Sigma}$ will represent a section of the cone $\mathbb{K}$ by the plane $w_{n}=1$. Through every point $\left(z_{1}, \ldots, z_{n-1}\right)=\left(w_{1}, \ldots, w_{n-1}\right)$ of the region $\bar{\Sigma}$ we lead a ray connecting this point with origin of coordinates. The set of all these rays forms the cone $\mathbb{K}$. (Any point of $\mathbb{K}$ is given by coordinates $\left(x z_{1}, \ldots, x z_{n-1}, x\right)$, where $x \geqslant 0$ and $\left(z_{1}, \ldots, z_{n-1}\right) \in \bar{\Sigma}$.)

Lemma 2. Let system (10) be a regular $\mathbb{R}$-system. If the region $\bar{\Sigma}$ is bounded, then $\mathbb{K}$ is an invariant cone with respect to system (10) and for any vector of initial values $\mathbf{w}(0) \in \mathbb{K}$ the solution $\mathbf{w}(t, \mathbf{w}(0))$ of system (10) is bounded.

Proof. We show that if $\mathbf{z}_{0}=\mathbf{z}(0) \in \bar{\Sigma}$, then $\forall t>0 \mathbf{z}(t) \in \bar{\Sigma}$. From here it will be follows that if $\mathbf{w}_{0}=\mathbf{w}(0) \in \mathbb{K}$, then $\forall t>0$ $\mathbf{w}(t) \in \mathbb{K}$.

Let $\mathbf{w}^{T}(t)=\left(w_{1}(t), \ldots, w_{n}(t)\right)$ be a bounded solution of system (10) such that $\lim _{t \rightarrow \infty} \mathbf{w}(t)=0$. Then using L'Hospital's rule we will have

$$
z_{i}^{*}=\lim _{t \rightarrow \infty} \frac{w_{i}(t)}{w_{n}(t)}=\lim _{t \rightarrow \infty} \frac{\dot{w}_{i}(t)}{\dot{w}_{n}(t)}=\lim _{t \rightarrow \infty} \frac{\mathbf{w}(t)^{T} D_{i} \mathbf{w}(t)}{\mathbf{w}(t)^{T} D_{n} \mathbf{w}(t)}, \quad i=1, \ldots, n-1 .
$$

Thus, if $\lim _{t \rightarrow \infty} w_{i}(t)=0$, then $\mathbf{z}^{* T}=\left(z_{1}^{*}, \ldots, z_{n-1}^{*}\right)$ is a root of system (12).

To investigate system (10), we will use the iterative Euler method:

$$
\mathbf{w}_{j+1}=\mathbf{w}_{j}-\left(\begin{array}{c}
\mathbf{w}_{j}^{T} D_{1} \mathbf{w}_{j} \\
\vdots \\
\mathbf{w}_{j}^{T} D_{n} \mathbf{w}_{j}
\end{array}\right) \Delta t, \quad j=0,1, \ldots,
$$

where $\mathbf{w}_{0}=\left(w_{10}, \ldots, w_{n 0}\right)^{T}$ and $\Delta t>0$ is a integration step.

Remind that $w_{n 0} \neq 0$. Then the vector

$$
\mathbf{z}_{0}=\left(z_{10}, \ldots, z_{n-1,0}\right)^{T}=\left(w_{10} / w_{n 0}, \ldots, w_{n-1,0} / w_{n 0}\right)^{T}
$$

is correctly defined. Let us apply also the iterative Euler method to system (11):

$$
\left\{\begin{array}{c}
\mathbf{z}_{j+1}=\mathbf{z}_{j}+\left(\begin{array}{c}
G_{1}\left(z_{1 j}, \ldots, z_{n-1, j}\right) w_{n j} \\
\vdots \\
G_{n-1}\left(z_{1 j}, \ldots, z_{n-1, j}\right) w_{n j}
\end{array}\right) \Delta t, \\
w_{n, j+1}=w_{n j}-\mathbf{w}_{j}^{T} D_{n} \mathbf{w}_{j} \Delta t, j=0,1, \ldots
\end{array}\right.
$$

Note that for the asymptotic stability of the trivial solution of system (11) it is necessary that the condition $\forall i w_{i 0}>0$ must be fulfilled.

Really, let $w_{n 0}<0$ and $\lim _{j \rightarrow \infty} \mathbf{w}_{j}=\mathbf{w}^{*}$, where $\left\|\mathbf{w}^{*}\right\|<\infty$. Then from (14) it follows that

$$
\left(\mathbf{w}^{*}\right)^{T} D_{1} \mathbf{w}^{*}=0, \ldots,\left(\mathbf{w}^{*}\right)^{T} D_{n} \mathbf{w}^{*}=0 .
$$

It means that forms $\mathbf{w}^{T} D_{1} \mathbf{w}, \ldots, \mathbf{w}^{T} D_{n} \mathbf{w}$ are linearly dependent (system (10) is not regular). We got the contradiction with Definition 2. Consequently it should be either $\left\|\mathbf{w}^{*}\right\|=0$ or $\left\|\mathbf{w}^{*}\right\|=\infty$. Its obvious that the case $\left\|\mathbf{w}^{*}\right\|=\infty$ always takes place if there exists a integer $k \in\{1, \ldots, n\}$ such that $w_{k 0}<0$. Thus, for the convergence of process (14) it must be $\forall i \in\{1, \ldots, n\}$ $w_{i 0} \geqslant 0$.

Now if $w_{n 0}=0$, then we have either

(a1) $-\mathbf{w}_{j}^{T} D_{n} \mathbf{w}_{j}<0$

or

(a2) $\forall i w_{n i}=w_{n 0}=0$, and $\mathbf{w}_{j}^{T} D_{n} \mathbf{w}_{j}=0$. 
It is clear that for some iteration under the number $k>0$ case (a1) reduces to the inequality $w_{n k}<0$ and therefore, we again have $\lim _{i \rightarrow \infty} w_{n i}=-\infty$. The case (a2) means that the vector of solutions $\mathbf{w}(t)=\left(w_{1}(t), \ldots, w_{n}(t) \equiv 0\right)^{T}$ must belong to some $(n-1)$-dimensional hyperplane in $\mathbb{R}^{n}$ which is defined by the equation $\left(w_{1}, \ldots, w_{n-1}\right) D\left(w_{1}, \ldots, w_{n-1}\right)^{T}=0$, where the matrix $D \in \mathbb{R}^{(n-1) \times(n-1)}$ is symmetrical. It means that in system (10) there are invariant subspaces and the last equation of system (10) has the form: $\dot{w}_{n}(t)=-\left(d_{n 1} w_{1}+\cdots+d_{n n} w_{n}\right) w_{n}$. It means the irregularity of system (10). As a result we again reduces to the contradiction.

Thus, $\forall i \in\{1, \ldots, n\}$, we have to have $w_{i 0}>0$. Then the vector $\mathbf{z}_{0}=\left(w_{10} / w_{n 0}, \ldots, w_{n-1,0} / w_{n 0}\right)^{T} \in \mathbb{R}^{n-1}$, all coordinates of which are positive numbers, can be constructed.

According to (15) we define vectors

$$
\mathbf{G}\left(\mathbf{z}_{i}\right)=\left(G_{1}\left(z_{1 i}, \ldots, z_{n-1, i}\right), \ldots, G_{n-1}\left(z_{1 i}, \ldots, z_{n-1, i}\right)\right)^{T} \in \mathbb{R}^{n-1}, \quad i=0,1, \ldots
$$

Let sets $\bar{\Sigma}_{+}$and $\bar{\Sigma}_{-}$be given by formulas

$$
\bar{\Sigma}_{+}=\bar{\Sigma}_{1} \cup \cdots \cup \bar{\Sigma}_{k}, \bar{\Sigma}_{-}=\bar{\Sigma}_{k+1} \cup \cdots \cup \bar{\Sigma}_{r},
$$

where without loss of generality we can consider that in domains $\bar{\Sigma}_{1}, \ldots, \bar{\Sigma}_{k}$

$$
G_{1}\left(z_{1}, \ldots, z_{n-1}\right) \geqslant 0
$$

and in domains $\bar{\Sigma}_{k+1}, \ldots, \bar{\Sigma}_{r}$

$$
G_{1}\left(z_{1}, \ldots, z_{n-1}\right) \leqslant 0 \text {. }
$$

(Thus, $\bar{\Sigma}=\bar{\Sigma}_{+} \cup \bar{\Sigma}_{-}, \Sigma_{+} \cap \Sigma_{-}=\emptyset$.)

By virtue of boundedness of domains $\bar{\Sigma}_{+}$and $\bar{\Sigma}_{-}$, we can define the finite magnitudes

$$
G_{1 \max }=\max _{\bar{\Sigma}_{+}} G_{1}\left(z_{1}, \ldots, z_{n-1}\right)>0, G_{1 \min }=\min _{\bar{\Sigma}_{-}} G_{1}\left(z_{1}, \ldots, z_{n-1}\right)<0 .
$$

Besides, we have

$$
\left.G_{i}\left(z_{1}, \ldots, z_{n-1}\right)\right|_{\mathbb{P}_{\mathrm{j}}}=0 ; i=1, \ldots, n-1 .
$$

(b) Assume that the point $\mathbf{z}_{0} \in \bar{\Sigma}_{+}$is an initial value of the iterative process $\mathbf{z}_{0}, \ldots, \mathbf{z}_{i}, \ldots$, which is defined by formulas (15).

Above it was shown that for convergence of iterative process (15) it is necessary that $w_{n i}>0$. We will assume that the last condition takes place. Then for the first coordinate of the vector $\mathbf{z}_{i}$, we have $z_{1 i}-z_{1, i-1}>0$. Thus, we obtain the monotone increasing sequence $z_{10}<z_{11}<\cdots<z_{1 m}<\cdots$ and for small enough $\Delta t$, we have $0<z_{1 m}-z_{1, m-1}$ $<w_{n, m-1} G_{1 \max } \Delta t<w_{n 0} G_{1 \max } \Delta t$.

(c) Now suppose that the point $\mathbf{z}_{0} \in \bar{\Sigma}_{-}$be an initial value of the iterative process $\mathbf{z}_{0}, \ldots, \mathbf{z}_{i}, \ldots$ which is defined by formulas (15). In this case we have $z_{1 i}-z_{1, i-1}<0$ and the sequence $z_{10}>z_{11}>\cdots>z_{1 k}>\cdots$ will be monotone decreasing and for small enough $\Delta t$ we have $z_{1, k-1}-z_{1 k}<-w_{n, k-1} G_{1 \min } \Delta t<-w_{n 0} G_{1 \min } \Delta t>0$ (remind that $w_{n j}>0$ ).

Regions $\bar{\Sigma}_{-}$and $\bar{\Sigma}_{+}$are defined by inequalities (16) and (17). By analogy with inequalities (16) and (17), the inequalities $G_{i}\left(z_{1}, \ldots, z_{n-1}\right) \leqslant 0$ (and $G_{i}\left(z_{1}, \ldots, z_{n-1}\right) \geqslant 0$ ) define regions $\bar{\Sigma}_{i+} \subset \bar{\Sigma}$ (and $\bar{\Sigma}_{i-} \subset \bar{\Sigma}$ ) which are analogously by the regions $\bar{\Sigma}_{-}$ and $\bar{\Sigma}_{+}$. (Thus, $\bar{\Sigma}=\bar{\Sigma}_{i+} \cup \bar{\Sigma}_{i-}, \Sigma_{i+} \cap \Sigma_{i-}=\emptyset$.) Therefore as in items (b) and (c), we can prove that $\forall q \in\{1, \ldots, n-1\}$ the sequence of coordinates with number $q z_{q 0}, z_{q 1}, \ldots, z_{q i}, \ldots$ for the vectors $\mathbf{z}_{0}, \mathbf{z}_{1}, \ldots, \mathbf{z}_{i}, \ldots$ is monotone and bounded. From here and (18) it follows that

$$
\liminf _{i \rightarrow \infty}\left\|\mathbf{z}_{i}-\mathbf{z}\right\|=0 ; \quad j=1, \ldots, n-1 .
$$

Hence $\forall i$ all coordinates of the vector $\mathbf{z}_{i}$ are positive, the sequence $\mathbf{z}_{0}, \mathbf{z}_{1}, \ldots, \mathbf{z}_{i}, \ldots \in \bar{\Sigma}$, and $\lim _{i \rightarrow \infty} \mathbf{z}_{i}=\mathbb{P}_{1} \cap \ldots \cap \mathbb{P}_{n-1}=\mathbf{z}_{k}^{*}$. Thus, the domain $\bar{\Sigma}$ (which is a closed set) is an invariant set. Besides, for small enough $\Delta t>0$ and $\Delta t \rightarrow 0$, we have $\mathbf{w}_{0}, \ldots, \mathbf{w}_{j}, \ldots \in \mathbb{K}$. Therefore the set $\mathbb{K}$ is invariant.

Lemma 3. Let system (10) be a regular $\mathbb{R}$-system. If the region $\bar{\Sigma}$ is bounded, then process (15) converges to the point $\mathbf{z}_{k}^{*}$; otherwise this process diverges.

Proof. For $n=2$ the first equation of system (11) has the form $\dot{z}_{1}(t)=\left(g_{11} z_{1}^{3}+\cdots+g_{14}\right) w_{2}(t)$, where $g_{11}, \ldots, g_{14}$ are real and $g_{11}>0$. Then system (12) consists on one equation $G\left(z_{1}\right)=g_{11} z_{1}^{3}+\cdots+g_{14}$.

Assume that the equation $G\left(z_{1}\right)=0$ has three real roots $z_{1}^{*}<z_{2}^{*}<z_{3}^{*}$. Then, $G\left(z_{1}\right)=g_{11}\left(z_{1}-z_{1}^{*}\right)\left(z_{1}-z_{2}^{*}\right)\left(z_{1}-z_{3}^{*}\right)$ and Jacobi's matrix of the function $G\left(z_{1}\right)$ is $L\left(z_{2}^{*}\right)=g_{11}\left(z_{2}^{*}-z_{1}^{*}\right)\left(z_{2}^{*}-z_{3}^{*}\right)<0$. Thus, the point $z_{2}^{*}$ is a stable and at $w_{20}>0$ process (15) is starting from bounded region $\left(z_{1}^{*}, z_{3}^{*}\right)$ converges (Fig. 2 ). 


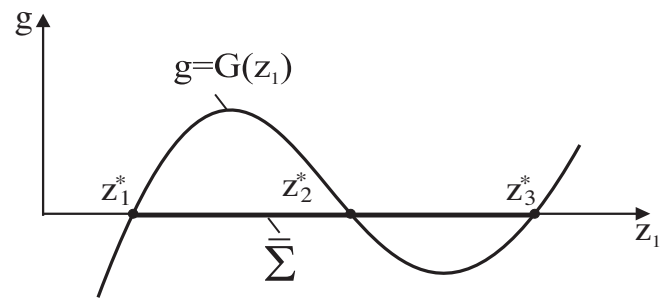

Fig. 2. The region $\bar{\Sigma}=\left[z_{1}^{*}, z_{3}^{*}\right]$.

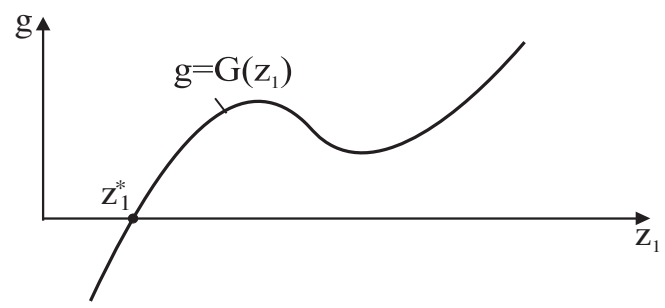

Fig. 3. The region $\bar{\Sigma}=z_{1}^{*}$ (the point $z_{1}^{*}$ is unstable).

Now we will assume that Eq. (12) has only one real root $z_{1}^{*}$. Then, $G\left(z_{1}\right)=g_{11}\left(z_{1}-z_{1}^{*}\right)\left(z_{1}^{2}+p z_{1}+q\right)$ and the polynomial $z_{1}^{2}+p z_{1}+q$ does not have real roots. From here it follows that $L\left(z_{1}^{*}\right)=g_{11} \cdot\left(\left(z_{1}^{*}\right)^{2}+p z_{1}^{*}+q\right)>0$. Therefore the point $z_{1}^{*}$ is unstable. It means that at $w_{20}>0$ iterative process (15) is starting from the unbounded region $\left(-\infty, z_{1}^{*}\right) \cup\left(z_{1}^{*}, \infty\right)$ will divergence (Fig. 3).

Now let $n>2$. That process (15) converges to the point $\mathbf{z}_{k}^{*} \in \bar{\Sigma}$ for the bounded region $\bar{\Sigma}$ it was proved in Lemma 2 . Introduce an $n$-dimensional real space $\mathbb{V}$ with coordinate axes $z_{1}, \ldots, z_{n-1}$, g. Let us consider an $n$-dimensional variety $\mathbb{G} \subset \mathbb{V}$ which defined by equations

$$
g=G_{1}\left(z_{1}, \ldots, z_{n-1}\right), \ldots, g=G_{n-1}\left(z_{1}, \ldots, z_{n-1}\right),
$$

where $\operatorname{deg} G_{i}\left(z_{1}, \ldots, z_{n-1}\right)=3$ with respect to $z_{i} ; i=1, \ldots, n-1$. (It is clear that a projection of $\mathbb{G}$ on the subspace $g=0$ contains the boundary of region $\bar{\Sigma}$.)

Denote by $\mathbb{H}$ any 2 -dimensional plane passing through the point $\mathbf{z}_{k}^{*}=\left(z_{k 1}^{*}, \ldots, z_{k, n-1}^{*}\right)^{T} ; k \in\{1, \ldots, l\} ; l \leqslant 2^{n}-1$, which is parallel by the axis $g$. Let

$$
\frac{z_{1}-z_{k 1}^{*}}{p_{1}}=\frac{z_{2}-z_{k 2}^{*}}{p_{2}}=\cdots=\frac{z_{n-1}-z_{k, n-1}^{*}}{p_{n-1}},
$$

be a system of equations generating this plane (here $p_{1}, p_{2}, \ldots, p_{n-1}$ are real parameters).

From system (20) it can obtained such equations:

$$
z_{1}=q_{1}\left(z_{j}\right), \ldots, z_{j-1}=q_{j-1}\left(z_{j}\right), z_{j+1}=q_{j+1}\left(z_{j}\right), \ldots, z_{n-1}=q_{n-1}\left(z_{j}\right),
$$

where $q_{1}\left(z_{j}\right), \ldots, q_{n-1}\left(z_{j}\right)$ are linear functions.

Intersect the variety $\mathbb{G}$ by the plane $\mathbb{H}$. System (19) consists on cubic equations. Therefore a projection of the intersection of $\mathbb{G}$ on coordinate plane $\left(z_{1}, g\right)$ will be presented by the cubic curve $g=G_{1}\left(z_{1}, \ldots, q_{n-1}\left(z_{1}\right)\right) \equiv b_{11}\left(p_{1}, \ldots, p_{n-1}\right) z_{1}^{3}$ $+b_{12}\left(p_{1}, \ldots, p_{n-1}\right) z_{1}^{2}+\cdots+b_{14}\left(p_{1}, \ldots, p_{n-1}\right)$, where $b_{11}\left(p_{1}, \ldots, p_{n-1}\right)>0$.

Similarly, using the system Eqs. (21), we will obtain the projections $g=b_{i 1}\left(p_{1}, \ldots, p_{n-1}\right) z_{j}^{3}+b_{i 2}\left(p_{1}, \ldots, p_{n-1}\right) z_{j}^{2}+\ldots+$ $b_{i 4}\left(p_{1}, \ldots, p_{n-1}\right)$ of the variety $\mathbb{G}$ on the coordinate plane $\left(z_{j}, g\right) ; b_{i 1}\left(p_{1}, \ldots, p_{n-1}\right)>0 ; i=2, \ldots, n-1 ; j=1, \ldots, n-1$.

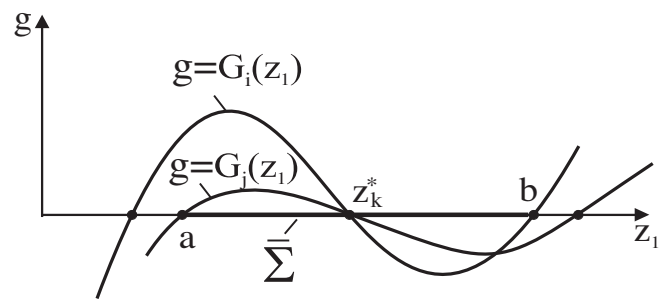

Fig. 4. The intersection of the region $\mathbb{G}$ by the plane $\mathbb{U}$ in case (d1). 
Generally there exist two forms of these projections.

(d1) For some $k$ there exist real parameters $p_{1}, \ldots, p_{n-1}$ such that $\forall i, j \in\{1, \ldots, n-1\}$ the equation $g=b_{i 1}\left(p_{1}, \ldots, p_{n-1}\right) z_{j}^{3}+b_{i 2}\left(p_{1}, \ldots, p_{n-1}\right) z_{j}^{2}+\cdots+b_{i 4}\left(p_{1}, \ldots, p_{n-1}\right)=0$ has three real roots (Fig. 4$)$ :

In this case the projection $\bar{\Sigma}$ on any plane $\left(z_{j}, g\right.$ ) (for example $\left(z_{1}, g\right)$ ) is equal $\bigcap_{n}^{n}\left[z_{i 1}^{*}, z_{i 3}^{*}\right]=[a, b] \neq \emptyset$. Therefore from Fig. 4 it follows that if an initial point $z_{0} \in\left(a, z_{k}^{*}\right) \subset \mathbb{M}$ or $z_{0} \in\left(z_{k}^{*}, b\right) \subset \mathbb{M}$, then the iterditive process (15) converges to the point $\mathbf{z}_{k}^{*}$. Thus, if the region $\bar{\Sigma}$ is bounded, then process (15) converges.

(d2) For any real parameters $p_{1}, \ldots, p_{n-1}, \forall k$, and $\forall i \in\{1, \ldots, n-1\}$ at least one of equations $g=b_{i 1}\left(p_{1}, \ldots, p_{n-1}\right) z_{j}^{3}+b_{i 2}\left(p_{1}, \ldots, p_{n-1}\right) z_{j}^{2}+\cdots+b_{i 4}\left(p_{1}, \ldots, p_{n-1}\right)=0$ has only one real root (Fig. 5):

In this case $\bar{\Sigma}=z_{k}^{*}$. Therefore from Fig. 5 it follows that if an initial point $z_{0} \in\left(-\infty, z_{k}^{*}\right) \subset \mathbb{U}$, then in the iterative process (15), we have $\lim _{i \rightarrow \infty} z_{i}=-\infty$. If $z_{0} \in\left(z_{k}^{*}, \infty\right) \subset \mathbb{U}$, then in the iterative process (15), we have $\lim _{i \rightarrow \infty} z_{i}=\infty$ and the iterative process (15) diverges.

Theorem 1. The regular $\mathbb{R}$-system (10) has the nontrivial asymptotic stability cone $\mathbb{K}$ if and only if the region $\bar{\Sigma}$ is bounded. In addition, if the number $l$ of positive solutions of system (12) is exactly equal $2^{n}-1$, then $\mathbb{K}$ always there exists.

Proof. Sufficiency. Let the region $\bar{\Sigma}=\bar{\Sigma}_{j_{1}} \cup \ldots \cup \bar{\Sigma}_{j_{r}}$ be bounded. The regions $\bar{\Sigma}_{j_{1}}, \ldots, \bar{\Sigma}_{j_{r}}$ have a unique point of intersection $\mathbf{z}_{k}^{*}$. Hence from Lemmas 2 and 3 it follows that $\mathbf{z}_{k}^{*}$ is attractor. This fact completes the proof of sufficiency.

Necessity. Assume that the region $\bar{\Sigma}$ is unbounded. Then from Lemma 3 it follows that at least for one coordinate $z_{i 0}$ $(i \in\{1, \ldots, n-1\})$ of the vector $\mathbf{z}_{0} \in \bar{\Sigma}$ the case (d2) takes place. Therefore for any vector of initial values $\mathbf{z}_{0}$ the iterative process (15) diverges. In other words, all coordinates of vectors $\mathbf{w}_{0}, \ldots, \mathbf{w}_{i}, \ldots$ in the iterative process (14) decrease unbounded: $\forall j \in\{1, \ldots, n\} \lim _{i \rightarrow \infty} w_{i j}=-\infty$. It completes the proof of necessity.

Now let $l=2^{n}-1$. Suppose that for any positive solution $\mathbf{z}_{i}^{*}, i=1, \ldots, l$, of system (12) the region $\bar{\Sigma}$ is unbounded. Then from the proof of Lemma 3 it follows that the case $(\mathrm{d} 2)$ takes place. It means that $l<2^{n}-1$. The obtained contradiction shows that for some $k \in\left\{1, \ldots, 2^{n}-1\right\}$ the region $\bar{\Sigma}$ have to be bounded. This completes the proof of Theorem 1 .

For system (11) we define the system

$$
\dot{\mathbf{z}}(t)=\mathbf{G}(\mathbf{z}(t))\left(\mathbf{G}=\left(G_{1}\left(z_{1}, \ldots, z_{n-1}\right), \ldots, G_{n-1}\left(z_{1}, \ldots, z_{n-1}\right)\right)^{T}\right),
$$

where $\mathbf{G}: \mathbb{R}^{n-1} \rightarrow \mathbb{R}^{n-1}$ is the same nonlinear operator that in (11).

Let

$$
L(\mathbf{z})=\left(\frac{\partial G_{i}(\mathbf{z})}{\partial z_{j}}\right) \in \mathbb{R}^{(n-1) \times(n-1)}, i, j=1, \ldots, n-1,
$$

be the Jacobi matrix of the vector-function $\mathbf{G}$. Denote by $\mathbf{z}_{1}^{*}, \ldots, \mathbf{z}_{l}^{*} \in \mathbb{R}^{n-1} ; 1 \leqslant l \leqslant 2^{n}-1$, all real solutions of system (12).

Theorem 2. The regular $\mathbb{R}$-system (10) has the nontrivial asymptotic stability cone $\mathbb{K}$ if and only if exactly one matrix from matrices $L\left(\mathbf{z}_{i}^{*}\right) ; i=1, \ldots, l$, is Hurwitz.

Proof. Necessity follows from the proof of Theorem 1. For system (22) an attractor point is the point $\mathbf{z}_{k}^{*}$. Therefore the matrix $L\left(\mathbf{z}_{k}^{*}\right)$ have to be Hurwitz. (See Theorem 2 [15].)

Sufficiency. Let the matrix $L\left(\mathbf{z}_{k}^{*}\right)$ be Hurwitz. We will take advantage of the proof of Lemma 3. Introduce the linear space $\mathbb{V}$ and the variety $\mathbb{G} \subset \mathbb{V}$. Then the projection of $\mathbb{G}$ onto subspace $g=0$ is $\bar{\Sigma}$ and $\mathbf{z}_{k}^{*} \in \bar{\Sigma}$. Intersect the variety $\mathbb{G}$ by the plane $\mathbb{U}$. Since the matrix $L\left(\mathbf{z}_{k}^{*}\right)$ is Hurwitz, then the point $\mathbf{z}_{k}^{*}$ is an attractor. Therefore a situation represented on Fig. 4 have to takes place (see case $(\mathrm{d} 1)$ ). It means that $\mathbb{K}$ is an asymptotic stability cone.

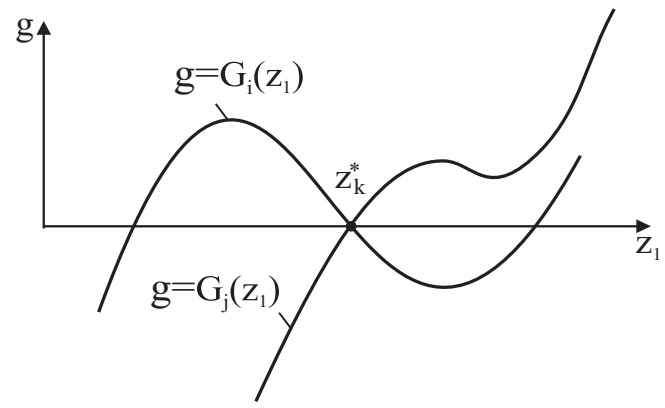

Fig. 5. The intersection of the region $\mathbb{G}$ by the plane $\mathbb{H}$ in case $(\mathrm{d} 2)$. 
Remark. It is clear that if Theorems 1 and 2 are valid for the variables $z_{1}, \ldots, z_{n-1}$, then they will be valid for variables $z_{1}=w_{1} / w_{i}, \ldots, z_{i-1}=w_{i-1} / w_{i}, z_{i}=w_{i+1} / w_{i}, \ldots, z_{n-1}=w_{n} / w_{i} ; i=1, \ldots, n-1$.

\section{Examples}

In this section the conditions of Theorem 1 are fulfilled.

Remark. Suppose that $n=2$ and for a regular system (9) system (12) has three real solutions. Then this condition is necessity and sufficient for existence an asymptotic stability cone (see Fig. 6).

4.1. Let $n=2$ and system (9) is represented as

$$
\left\{\begin{array}{l}
\dot{w}_{1}=-\left(-w_{1}+0.8 w_{2}\right)^{2} \\
\dot{w}_{2}=-\left(0.8 w_{1}-w_{2}\right)^{2} .
\end{array}\right.
$$

4.2. Let $n=3$ and system (9) has the form [15]

$$
\left\{\begin{array}{l}
\dot{w}_{1}=-\left(-2 w_{1}+w_{2}+w_{3}\right)^{2} \\
\dot{w}_{2}=-\left(2 w_{1}-3 w_{2}+w_{3}\right)^{2} \\
\dot{w}_{3}=-\left(w_{1}+w_{2}-3 w_{3}\right)^{2}
\end{array}\right.
$$

In Fig. 7 the digits $2,3,4,6$, and 7 represent respectively the roots $z_{2}^{*}, z_{3}^{*}, z_{4}^{*}, z_{6}^{*}$, and $z_{7}^{*}$ of system (12). (The roots $z_{1}^{*}$, and $z_{5}^{*}$ are not included in the graph.) The cone $\mathbb{K}$ is shown on Fig. 8.

\section{Stability cones for inhomogeneous systems}

The main result of this section contains in the following theorem.

Theorem 3. Let $\mathbb{K}$ be the nontrivial asymptotic stability cone for the regular system (9) and let the vector $\mathbf{k}=\left(k_{1}, \ldots, k_{n}\right)^{T} \in \mathbb{K}$. Then for system (8) $\mathbb{K}$ is a stability cone.

Proof. Again apply the iterative Euler method for the investigation of solutions of system (8):

$$
\mathbf{w}_{i+1}=\mathbf{w}_{i}+\frac{1}{4} \mathbf{k} \cdot \Delta t-\left(\begin{array}{c}
\left(c_{11} w_{1 i}+\cdots+c_{1 n} w_{n i}\right)^{2} \\
\vdots \\
\left(c_{n 1} w_{1 i}+\cdots+c_{n n} w_{n i}\right)^{2}
\end{array}\right) \cdot \Delta t, \quad i=0,1, \ldots,
$$

where $\mathbf{w}_{0} \in \mathbb{K}$ is a vector of initial values; $\Delta t>0$ is a sufficiently small number.

According to the conditions of Theorem $3 \mathbb{k}$ is the nontrivial asymptotic stability cone for system (9). Then from condition $\mathbf{w}_{0} \in \mathbb{K}$ it follows that the vector

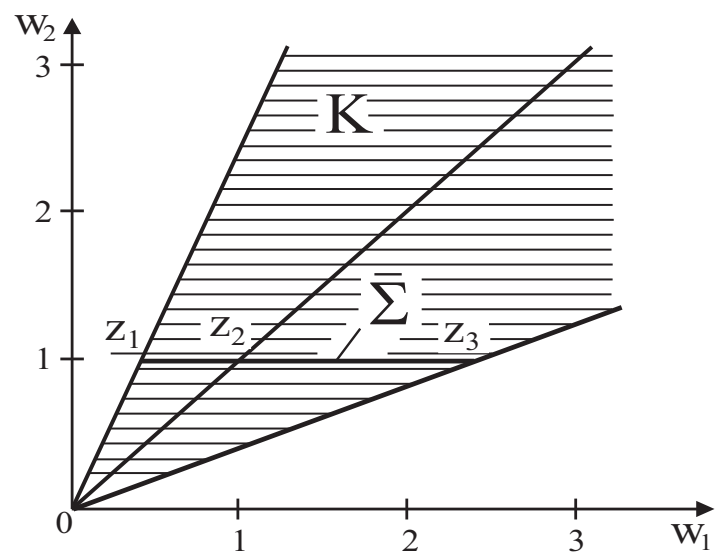

Fig. 6. The asymptotic stability cone $\mathbb{K}$ and the domain of asymptotic stability $\bar{\Sigma}$ for system (23). 


$$
\mathbf{h}_{1}=\mathbf{w}_{0}-\left(\begin{array}{c}
\left(c_{11} w_{10}+\cdots+c_{1 n} w_{n 0}\right)^{2} \\
\vdots \\
\left(c_{n 1} w_{10}+\cdots+c_{n n} w_{n 0}\right)^{2}
\end{array}\right) \cdot \Delta t \in \mathbb{K} .
$$

Then by virtue of the condition $\mathbf{k} \in \mathbb{K}$ for a sufficiently small number $\Delta t>0$, we have $\mathbf{w}_{1}=\mathbf{h}_{1}+\mathbf{k} \cdot(\Delta t) / 4 \in \mathbb{K}$.

From the iterative Euler method (25) for $i=1,2, \ldots$, we derive that $\forall i \mathbf{w}_{i} \in \mathbb{K}$. In other words, the cone $\mathbb{K}$ is an invariant set for system (8) (if $\mathbf{w}_{0} \in \mathbb{K}$, then $\forall t>0 \mathbf{w}(t) \in \mathbb{K}$ ).

Now we will show that $\mathbb{K}$ is a stability cone. Since $\forall t \geqslant 0 \mathbf{w}(t) \in \mathbb{K} \subset \mathbb{R}^{n+}$, then solutions $w_{i}(t), i=1, \ldots, n$ are bounded below by number 0 . Hence we have $w_{i}(t) \geqslant 0, i=1, \ldots, n$. Besides, from condition $\forall t \geqslant 0 \mathbf{w}(t) \in \mathbb{K}$ it also follows that there does not exist a point $a>0$ such that $\lim _{t \rightarrow a}\|\mathbf{w}(t)\|=\infty$.

Suppose that $\exists j \in\{1, \ldots, n\}$ such that $\lim _{t \rightarrow \infty} w_{j}(t)=\infty$ (the solution $w_{j}(t)$ is unbounded). Then by virtue of the regularity system (8), $\forall i \in\{1, \ldots, n\} \lim _{t \rightarrow \infty} w_{i}(t)=\infty$ (all solutions are unbounded). The last limit equality is possible only in the case if $\exists t^{*}>0$ such that for $t>t^{*}$

$$
0.25 k_{i}-\left(c_{i 1} w_{1}(t)+\cdots+c_{i n} w_{n}(t)\right)^{2} \geqslant 0, i=1, \ldots, n .
$$

Summing of inequalities (26), we get (at $t>t^{*}$ ) the inequality

$$
\sum_{i=1}^{n} \sum_{j=1}^{n} p_{i j} w_{i}(t) w_{j}(t) \leqslant d
$$

where $d>0$.

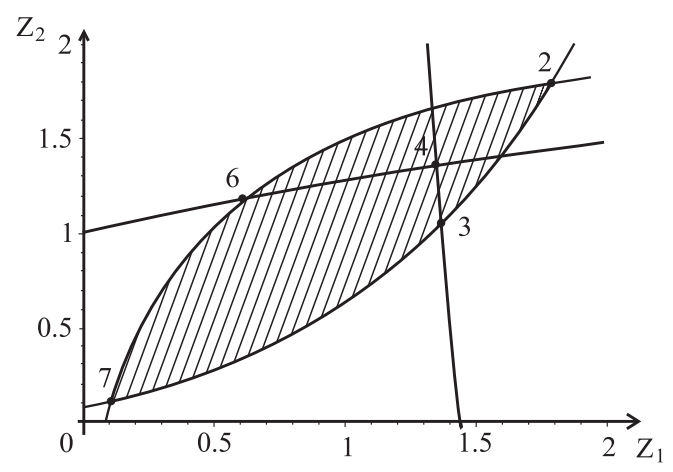

Fig. 7. The domain of asymptotic stability $\bar{\Sigma}$ for system (24).

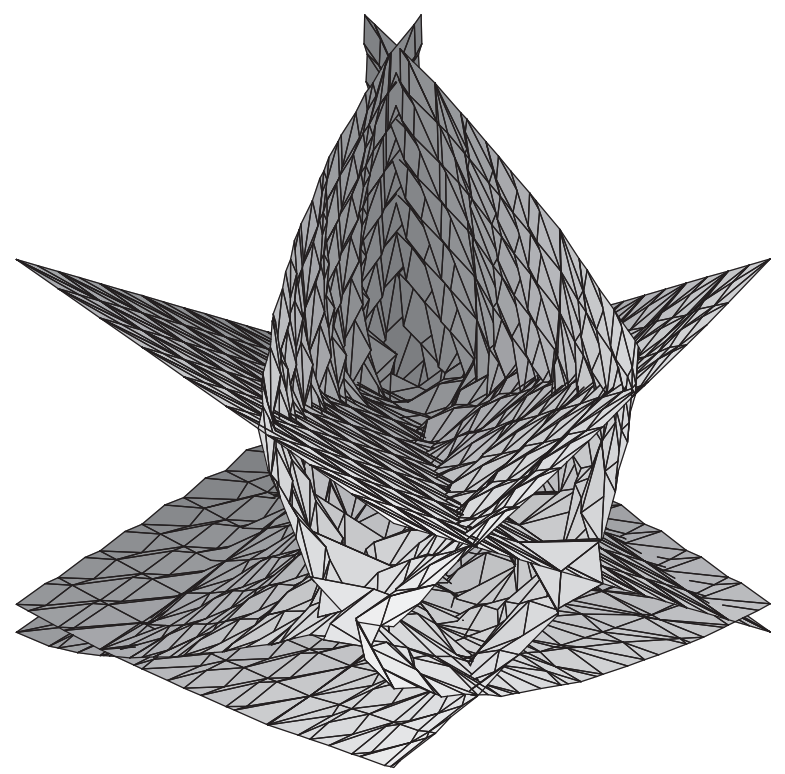

Fig. 8. The asymptotic stability cone $\mathbb{K}$ for system (24) (the cross-section of this cone is shown in Fig. 7). 
Further, by virtue of regularity of system (8), the quadratic form located in the left-hand side of inequality (27) is positive definite. Consequently, at $t>t^{*}$ all solutions of system (8), it is situated in a closed ellipsoid which is defined by inequality (27). Therefore all these solutions are bounded. It contradiction completes the proof. (It should be said that if all solutions $w_{i}(t)$ are limited, then coordinates $\left(w_{1}^{*}, \ldots, w_{n}^{*}\right)$ of a limit point are solutions of the system of algebraic equations: $0.25 k_{i}-\left(c_{i 1} w_{1}+\cdots+c_{i n} w_{n}\right)^{2}=0, i=1, \ldots, n$. $)$

Remark. Theorem 3 is trivially generalized on systems (10).

We will return to the initial base of the space $\mathbb{R}^{n}$. Then system (8) will return to system (2) and cone $\mathbb{K}$ will return to some cone $\mathbb{K}_{N}$, the vertex of which already does not coincide with origin of coordinates. From the proof given above it follows that this cone will be an invariant stability cone for system (2). Notice that the vertex of cone $\mathbb{K}_{N}$ is the point $N=\left(k_{1} / 2, \ldots, k_{n} / 2\right)$.

\subsection{Consider the following example. Assume that system (2) has the form}

$$
\left\{\begin{array}{l}
\dot{x}_{1}=x_{1}\left(1-x_{1}\right)-0.8 x_{2}\left(1-x_{2}\right) \\
\dot{x}_{2}=-0.8 x_{1}\left(1-x_{1}\right)+x_{2}\left(1-x_{2}\right) .
\end{array}\right.
$$

For system (28) we write system (8) as

$$
\dot{w}_{1}=0.25-\left(w_{1}-0.8 w_{2}\right)^{2}, \quad \dot{w}_{2}=0.25-\left(-0.8 w_{1}+w_{2}\right)^{2} .
$$

On next figures typical phase portraits and stability cones are represented. For system (28) the vertex of the stability cone is the point $N(0.5 ; 0.5)$ (Fig. 9), and for system (29) the vertex of the stability cone coincides with origin of coordinates. We mark that the asymptotic stability cone for the homogeneous system (23) (Fig. 6) completely coincides with the cone for system (29) (Fig. 10).

\subsection{Consider the following system}

$$
\left\{\begin{array}{l}
\dot{x}_{1}=7 x_{1}\left(1-x_{1} / k_{1}\right)+6 x_{2}\left(1-x_{2} / k_{2}\right) \\
\dot{x}_{2}=1 x_{1}\left(1-x_{1} / k_{1}\right)+12 x_{2}\left(1-x_{2} / k_{2}\right) .
\end{array}\right.
$$

Here the parameters $k_{1}, k_{2}$ are supposed by unknown controls.

For system (30) system (8) has the form

$$
\dot{w}_{1}=0.25 k_{1}-\left(7 w_{1}+6 w_{2}\right)^{2} / k_{1}, \quad \dot{w}_{2}=0.25 k_{2}-\left(w_{1}+12 w_{2}\right)^{2} / k_{2} .
$$

For system (30) Eq. (12) may be written as

$$
\lambda \cdot z(z+12)^{2}-(7 z+6)^{2}=0, \quad \lambda=k_{1} / k_{2}, \quad z=w_{1} / w_{2} .
$$

We will gradually increase $\lambda$ beginning with 0 . A process is stopped, when Eq. (31) will be to have three positive roots. In accordance with Theorem 1 it means that at given $\lambda$ system (30) has the asymptotic stability cone.

Here $\lambda=1.2$. Then we can take $k_{1}=6, k_{2}=5$. In this case Eq. ( 31 ) has three positive roots: $z_{1}=0.45 ; z_{2}=7.50 ; z_{3}=8.88$. (We chose $\lambda=1.2$ so that $z_{1}<\lambda<z_{3}$. In this case the conditions of Theorem 3 are fulfilled.).

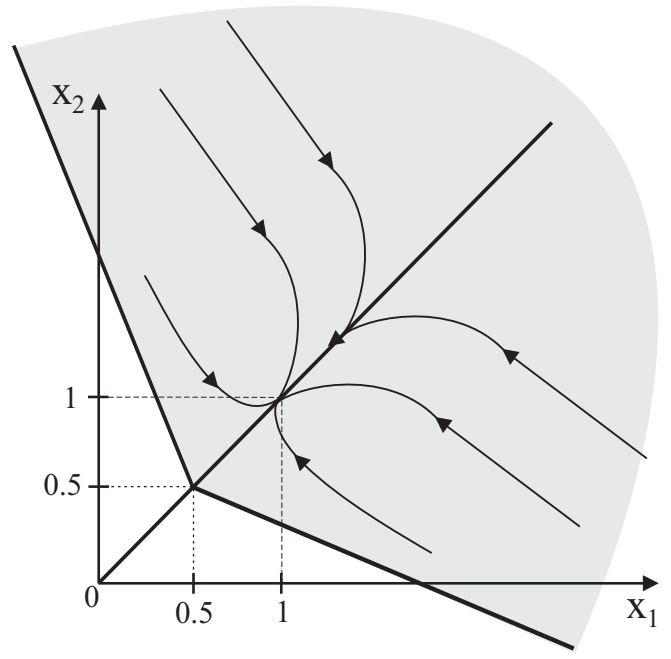

Fig. 9. The phase portrait and the stability cone for system (28). 


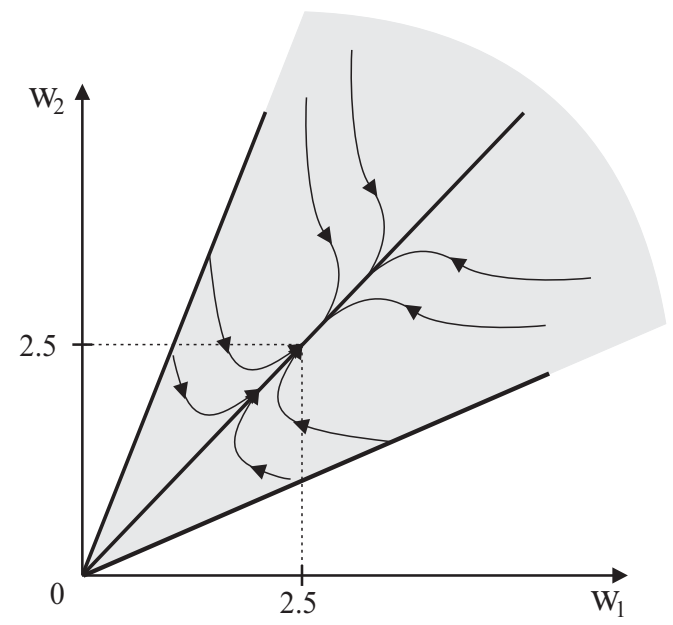

Fig. 10. The phase portrait and the stability cone for system (29).

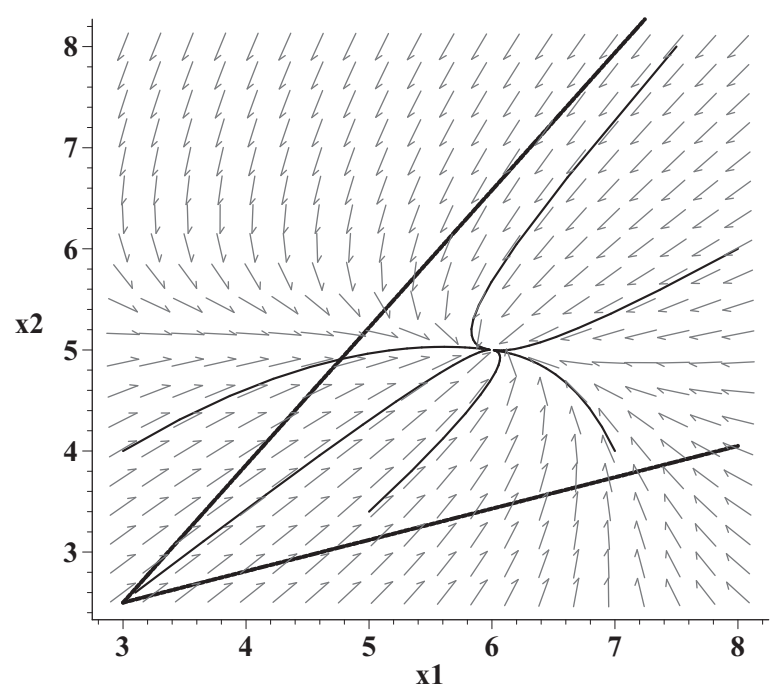

Fig. 11. The phase portrait and the stability cone for system (30).

Let us get back to variables $x_{1}, x_{2}$. Then boundaries of the stability cone (it are rays) are defined by equations

$$
\begin{aligned}
& x_{2}=\frac{a_{21} z_{1}+a_{22}}{a_{11} z_{1}+a_{12}} x_{1}-\frac{a_{21} z_{1}+a_{22}}{a_{11} z_{1}+a_{12}}+\frac{k_{2}}{2}=1.36 x_{1}-1.58, \\
& x_{2}=\frac{a_{21} z_{3}+a_{22}}{a_{11} z_{3}+a_{12}} x_{1}-\frac{a_{21} z_{3}+a_{22}}{a_{11} z_{3}+a_{12}}+\frac{k_{2}}{2}=0.31 x_{1}+1.57 .
\end{aligned}
$$

The vertex of this cone is the point $N=(3 ; 2.5)$ (see Fig. 11).

\section{Conclusion}

The generalization of results of paper [15], which allowed not only to establish the existence of stability cones for some types of the quadratic systems, but also describe their forms, is offered in the present paper. In addition, Theorems 1-3 can be used not only for analysis tasks, but also and for design problems. This fact was used for research of model (2). In this model magnitudes $a_{i j}$ describe the biological features of sub-populations. At the same time magnitudes $k_{i}$ determining sizes of ecological niches can be considered as controls. In Example 4.2 it is shown as possible to construct the constant controls $k_{i}=f_{i}\left(a_{i j}\right) ; i, j=1,2$, in order that to provide the coexistence of biological sub-populations $x_{1}, x_{2}$ are described by model (2).

\section{References}

[1] G. Rowe, Theoretical Models in Biology, Springer, New York, 1994.

[2] H.T. Odum, Ecological and General Systems and Introduction to Systems Ecology, University Press of Colorado, Niwot, 1994. 
[3] M. Williamson, The Analysis of Biological Populations, L. Academic Press, New York, 1990.

[4] E.C. Pielou, Mathematical Ecology, Wiley and Sons, New York, 1977.

[5] I. Gubelj, K.A.J. White, Spatial heterogeneity, social structure and disease dynamics of animal populations, Theor. Popul. Biol. 66 (2) (2004) 139-149.

[6] C.M. Breder, Studies on social grouping in fishes, Bull. Am. Museum Nat. Hist. 117 (6) (1959) 397-481.

[7] D. Croft, R. James, J. Krause, Exploring Animal Social Networks, Prinston University Press, Prinston, 2009.

[8] J.C. Poggiale, From behavioral to population level: growth and competition, Math. Comput. Model. 27 (4) (1998) 41-49.

[9] M. Franz, C.I. Nunn, Social structure in colonial mammal: unravelling hidden structural layers and their foundation by network analysis, Anim. Behav. 74 (5) (2007) 1293-1302.

[10] B.A. Maurer, Untangling Ecological Complexity, Univ. of Chicago Press, Chicago, 1999.

[11] Y. Takeuchi, Global Dynamical Properties of Lotka-Volterra Systems, World Scientific, Singapore, 1996.

[12] S.V. Chernyshenko, Nonlinear Analysis of Forest Ecosystems Dynamics, Dnipropetrovsk University Press, Dnipropetrovsk, 2005.

[13] S. Karlin, Some mathematical models of population genetics, Am. Math. Mon. 79 (7) (1972) 699-739.

[14] V.Ye. Belozyorov, Design of linear feedback for bilinear control systems, Int. J. Appl. Math. Comput. Sci. 12 (4) (2002) $493-511$.

[15] V.Ye. Belozyorov, On stability cones for quadratic systems of differential equations, J. Dyn. Control Syst. 11 (3) (2005) $329-351$.

[16] V.Ye. Belozyorov, Invariant approach to an existence problem of nontrivial asymptotic stability cone, Canadien Appl. Math. Q. 15 (2) (2007) 125-168.

[17] D. Mumford, Algebraic Geometry. Complex Projective Varieties, Springer-Verlag, Berlin, Heidelberg, New York, 1976. 